\title{
Windenergie und Grundwasser
}

\section{Nico Goldscheider ${ }^{1}$}

Angenommen: 13. September 2021 / Online publiziert: 30. September 2021

(c) Der/die Autor(en) 2021

Aktuell stehen in Deutschland rund 30.000 Windenergieanlagen (WEA). Im Zuge der Energiewende soll sich diese Zahl bis 2050 erheblich vergrößern, wobei sehr unterschiedliche Zahlen kursieren: Häufig genannte Prognosen gehen von dann insgesamt 37.000-65.000 WEA aus. Nach anderen Schätzungen wären insgesamt rund 300.000 WEA notwendig, um die ausfallenden Kapazitäten von Kernenergie und fossilen Energieträgern zu kompensieren und gleichzeitig den Umstieg auf Elektromobilität zu ermöglichen, also deutschlandweit fast eine WEA pro Quadratkilometer, womit aber das Problem der fehlenden Grundlastfähigkeit immer noch nicht gelöst wäre. Allein in Baden-Württemberg sollen durch eine „Vergabeoffensive für die Vermarktung von Staatswald- und Landesflächen für die Windkraftnutzung" bis zu 1000 neue WEA entstehen.

Es ist davon auszugehen, dass an vielen Standorten mit hoher Windhöffigkeit und geringem Konfliktpotenzial bereits heute eine WEA steht. Entsprechend ist beim zukünftigen Ausbau der Windenergie mit zunehmenden Konflikten zu rechnen. Neben visuellen und akustischen Belastungen und dem Artenschutz - allein rund 15.000 Mäusebussarde und 250.000 Fledermäuse werden jedes Jahr in Deutschland an WEA getötet -, rückt auch das Konfliktpotenzial zwischen Windenergie und Grundwasser immer häufiger in den Fokus; zum einen, weil Bürgerinitiativen jede Möglichkeit suchen, um den Bau weiterer WEA zu verhindern; zum anderen aber auch, weil WEA gerade in den Mittelgebirgen oftmals in unmittelbarer Nähe von genutzten Quellen oder grundwasserabhängigen Ökosystemen geplant werden.

Beispielsweise gab und gibt es im Schwarzwald mehrere Windenergie-Projekte, bei denen der Grundwasserschutz im Zentrum des Interesses stand und teils zu einer Veränderung der Planung oder zu einer Ablehnung des Projektes führte. Bei einem dieser Projekte waren fünf WEA

Nico Goldscheider

nico.goldscheider@kit.edu

1 Institut für Angew. Geowissenschaften, Karlsruher Institut für Technologie, Kaiserstr. 12, 76131 Karlsruhe, Deutschland im erweiterten Einzugsbereich der Quellen einer Brauerei geplant, die mit der Reinheit ihres Brauwassers und unberührter Natur wirbt; hier wurde die Zahl der geplanten Anlagen reduziert und die Abstände zu den Brauwasserquellen erhöht. In einem anderen Fall ging es um mehrere WEA im Gipfelbereich eines Berges, an dessen Fuß die Trinkwasserquellen einer Kleinstadt liegen; hier wurden die WEA in einer Volksabstimmung abgelehnt. Ein interessanter Unterschied bestand darin, dass für die Quellen der privaten Brauerei keine Schutzzonen existierten, während die öffentlichen Quellen über solche verfügten. Die zuständigen Behörden haben jedoch entschieden, dass der Schutz der Brauereiquellen trotz fehlender Schutzzonen ebenso gewährleistet sein muss.

Bei der systematischen Bewertung des Konfliktpotenzials zwischen Windenergie und Grundwasser muss zwischen den Aspekten Wassermenge und Wasserqualität und zwischen der Bauphase und dem laufenden Betrieb unterschieden werden. Eine Analyse der damit verbundenen Befürchtungen und Risiken ergibt in den meisten Fällen, dass Sorgen hinsichtlich der Wassermenge, bis hin zu einem Versiegen von Quellen, unbegründet sind, außer in sehr speziellen hydrogeologischen und bautechnischen Situationen. Anders sieht es mit den Sorgen rund um die Wasserqualität aus, insbesondere während der Bauphase, denn die Errichtung einer WEA ist mit einer erheblichen Baustelle verbunden. Allein das Fundament besteht aus über $1000 \mathrm{~m}^{3}$ Beton und erfordert eine entsprechend große Baugrube. In vielen Fällen ist auch der Bau oder Ausbau einer Zufahrtsstraße notwendig, mit allen damit verbundenen Risiken, wie umfangreiche Erdarbeiten, intensiver Lkw-Verkehr und die Gefahr auslaufender Kraftstoffe und sonstiger wassergefährdender Stoffe. Auch wenn all diese Gefahren beherrschbar erscheinen, sind sie durchaus relevant, denn es geht um eine große Anzahl von WEA, und viele geplante Standorte befinden sich in verletzlichen Gebirgslandschaften, in unmittelbarer Nähe von genutzten Quellen und grundwasserabhängigen Ökosystemen. Etwas anders sieht es im Flachland aus, wo WEA meist auf landwirtschaftlichen Flächen errichtet werden und den Landwirten langfristige Einnahmen sichern. 
Teils sind Trinkwasserversorger gleichzeitig auch Betreiber oder Anteilseigner von Windkraftanlagen; in diesen Fällen wird aus dem Konfliktpotenzial zumindest ökonomisch eine Interessensgleichheit.

Wesentlich komplexer und weniger gut erforscht sind die Auswirkungen von WEA auf das Klima. Da WEA den Wind ausbremsen und der Atmosphäre Energie entziehen, beeinflussen sie auch den Wasserdampftransport und das Temperaturfeld. Bei der Bewertung einzelner Standorte dürfte dies kaum eine Rolle spielen, wohl aber bei der Technikfolgenabschätzung des Ausbaus der Windenergie im großen Stil. Hier besteht Forschungsbedarf, auch hinsichtlich der Auswirkungen auf die Bodenfeuchte, Abflüsse und Grundwasserneubildung.

Die zuständigen Landesämter und Ministerien haben zu diesem Themenfeld relevante Leitfäden, Richtlinien und Erlasse verfasst. Aus der Sicht der Hydrogeologie und Wasserwirtschaft war die Windenergie bisher kein großes Thema. Beim Ausbau derselben rücken aber Konflikte rund ums Grundwasser immer häufiger in den Fokus. Daher wäre es sinnvoll, wenn sich die Hydrogeologie intensiver mit dieser Thematik auseinandersetzen würde. Aufgrund der geringen Energieausbeute pro Fläche führt der vollständige Umstieg auf erneuerbare Energien generell zu einem größeren Flächenbedarf für die Energiegewinnung, sodass die Konflikte mit Mensch und Umwelt weiter zunehmen werden, wobei auch die Grundwasserressourcen oftmals eine wichtige Rolle spielen.

Funding Open Access funding enabled and organized by Projekt DEAL.

Open Access Dieser Artikel wird unter der Creative Commons Namensnennung 4.0 International Lizenz veröffentlicht, welche die Nutzung, Vervielfältigung, Bearbeitung, Verbreitung und Wiedergabe in jeglichem Medium und Format erlaubt, sofern Sie den/die ursprünglichen Autor(en) und die Quelle ordnungsgemäß nennen, einen Link zur Creative Commons Lizenz beifügen und angeben, ob Änderungen vorgenommen wurden.

Die in diesem Artikel enthaltenen Bilder und sonstiges Drittmaterial unterliegen ebenfalls der genannten Creative Commons Lizenz, sofern sich aus der Abbildungslegende nichts anderes ergibt. Sofern das betreffende Material nicht unter der genannten Creative Commons Lizenz steht und die betreffende Handlung nicht nach gesetzlichen Vorschriften erlaubt ist, ist für die oben aufgeführten Weiterverwendungen des Materials die Einwilligung des jeweiligen Rechteinhabers einzuholen.

Weitere Details zur Lizenz entnehmen Sie bitte der Lizenzinformation auf http://creativecommons.org/licenses/by/4.0/deed.de. 